\title{
An Efficient Dynamic Programming Algorithm and Implementation for RNA Secondary Structure Prediction
}

\author{
Guangming Tan, Xinchun Liu, and Ninghui Sun \\ Institutue of Computing Technology, Chinese Academy of Sciences, China \\ \{tgm, lxc, snh\}@ncic.ac.cn
}

\begin{abstract}
RNA secondary structure prediction based on free energy rules for stacking and loop conformation remains a major computational method. The basic dynamic programming algorithm needs $\mathrm{O}\left(\mathrm{n}^{4}\right)$ time to calculate the minimum free energy for RNA secondary structure. To date, there are two variants for handling this problem: either the internal loops are bounded by a maximal size $\mathrm{k}$ giving a time complexity of $\mathrm{O}\left(\mathrm{n}^{2} * \mathrm{k}^{2}\right)$, or one uses the trick of Rune Lyngso, which makes use of the regularities of loop energies, to reduce time complexity to $\mathrm{O}\left(\mathrm{n}^{3}\right)$ without restriction. We propose a new dynamic programming algorithm for RNA secondary structure prediction by analyzing energy rules. Through only additional $\mathrm{O}(\mathrm{n})$ space, this algorithm eliminates redundant calculation in the energy calculation of internal loop with unrestricted/restricted size and reduces the time complexity of this part from $\mathrm{O}\left(\mathrm{n}^{4}\right)$ to $\mathrm{O}\left(\mathrm{n}^{3}\right)$, then the overall time complexity to $\mathrm{O}\left(\mathrm{n}^{3}\right)$.
\end{abstract}

\section{Introduction}

RNA secondary structure prediction has been one of major research area of computational biology. Many computational methods have been proposed since experimental methods determining secondary structure are time and cost consuming. Tinoco et.al. (1971, 1973) proposed a good approximate method to calculate the stability of a folded RNA molecule by adding independent contributions from base pair stacking and loop-destabilizing terms from secondary structure. Based on this model, algorithms for computing the most stable structures have been proposed. Dynamic programming algorithms (Zuker et al, 1989) based on the minimum free energy model are practical methods for RNA secondary structure prediction without pseudo-knots. They give rigorous solutions to the problems of computing a minimum free energy structure.

In general, the dynamic programming algorithms require $\mathrm{O}\left(\mathrm{n}^{4}\right)$ time (where $\mathrm{n}$ is the length of RNA sequence), especially for the internal loop. If the stability of an internal loop can be assumed only to depend on the size of internal loop, Waterman and Smith(1986) describe how to reduce the time requirement to $\mathrm{O}\left(\mathrm{n}^{3}\right)$. By bounding internal loops to small loops (k), a method risks missing some optimal large internal loops, but the time required for evaluating internal loops is reduced to $\mathrm{O}\left(\mathrm{k}^{2}\right)$, thus reducing the overall complexity to $\mathrm{O}\left(\mathrm{k}^{2} \mathrm{n}^{2}\right)$ ( Hofacker, Ivo L. 1994, 2003). For unrestricted internal loop, a $\mathrm{O}\left(\mathrm{n}^{3}\right)$ algorithm was proposed by Rune Lyngso et 
al(1999). This algorithm allows all internal loops to be evaluated in time $\mathrm{O}\left(\mathrm{n}^{3}\right)$ based on a property of current free energy functions.

In this paper, based on the regularities of loop energies, we will propose an efficient dynamic programming algorithm that also reduces the overall time complexity to $\mathrm{O}\left(\mathrm{n}^{3}\right)$, but with only $\mathrm{O}(\mathrm{n})$ additional space. The rest of this paper is structured as follows. In METHODS, we briefly describe the problem of evaluating energies of loops in RNA secondary structure prediction. In ALGORITHMS, we present an efficient $\mathrm{O}\left(\mathrm{n}^{3}\right)$ algorithms for calculating energies for certain free energy functions. In IMPLEMENTATION, we compare our method to the popular previously used method. CONCLUSION concludes this paper.

\section{Methods}

The formal definition of an RNA secondary structure is described by Rune Lyngso et.al and we will use the same definition and notations (In the rest of this paper, we will not give the definition and the explanation of notations since we refer to this article for a presentation of the dynamic programming algorithm). The secondary structure is assumed not to contain pseudo-knots. One can view a pseudo-knot free secondary structure $\mathbf{S}$ as a collection of loops together with some external unpaired bases. The loops are classified into six kinds of loops: hairpin loop, bulge, internal loop, multi-branched loop and stacked pair.

The energy of an RNA secondary structure is assumed to be the sum of the energies of the independent loops of the structure, that is, the energy of a loop only depends on the loop and not on the rest of the structure (Tinoco et al 1973). Based on these assumptions one can specify a recursion to calculate the energy of the optimal structure for a sequence $s$. The energy of an optimal structure of the sequence from $\mathrm{i}$ through $\mathrm{j}$ closed by $(\mathrm{i}, \mathrm{j})$ :

$$
V(i, j)=\min \{e H(i, j), e S(i, j)+V(i+1, j-1), V B I(i, j), V M(i, j)\}
$$

Where eH(i,j) is the energy of a hairpin loop closed by $(i, j)$ and eS(i,j) is the energy of stacking base pair $(i, j)$ with $(i+1, j-1)$. The energy VBI $(i, j)$ of an internal loop closed by $(\mathrm{i}, \mathrm{j})$ :

$$
V B I(i, j)=\min _{\substack{i<i^{\prime}<j^{\prime}<j \\ i^{\prime}-i+j-j^{\prime}>2}}\left\{e L\left(i, j, i^{\prime}, j^{\prime}\right)+V\left(i^{\prime}, j^{\prime}\right)\right\}
$$

$\operatorname{VM}(\mathrm{i}, \mathrm{j})$ is the energy of multi-branched loop closed by $(\mathrm{i}, \mathrm{j})$ :

$$
V M(i, j)=\min \left\{\begin{array}{l}
\min _{i+1<<j-1}\{W M(i+1, k-1)+W M(k, j-1)\} \\
\min _{i+2<<j-1}\{W M(i+2, k)+W M(k+1, j-1)\}+d B \\
\min _{i+1<<<j-2}\{W M(i+1, k)+W M(k+1, j-2)\}+d 5 \\
\min _{i+2<<j-2}\{W M(i+2, k)+W M(k+1, j-2)\}+d 3+d 5
\end{array}\right\}
$$


Where $\mathrm{d} 3$ and $\mathrm{d} 5$ is the energy of dangle on $3^{\prime}$ and $5^{\prime}, \mathrm{WM}(\mathrm{i}, \mathrm{j})$ is an extra array, it can be calculated from:

$$
\begin{gathered}
W M(i, j)=\min \{V(i, j)+b, W M(i, j-1)+c, W M(i+1, j)+c \\
\left.\min _{i<k \leq j}\{W M(i, k-1)+W M(k, j)\}\right\}
\end{gathered}
$$

From the four equations above, we can see that the dynamic programming algorithm should fill some arrays. Equation 2 shows that the evaluation of internal loops needs $\mathrm{O}\left(\mathrm{n}^{4}\right)$ time. Equation 3 and 4 show that the evaluation of multi-branched loops need $\mathrm{O}\left(\mathrm{n}^{3}\right)$ time. The overall time complexity of the dynamic programming algorithm is $\mathrm{O}\left(\mathrm{n}^{4}\right)$. In the next section, based on the property of current free energy functions discovered by Rune Lyngso et.al, an efficient algorithm to evaluate the internal loops with unrestricted size will be proposed. Furthermore, through eliminating redundant calculation, our method speedups original algorithm when the maximum size of internal loops is restricted to MAXLOOP.

\section{Algorithms}

In order to calculate $\operatorname{VBI}(\mathrm{i}, \mathrm{j})$, the algorithm should calculate the minimum energy among all base pairs (i',j'), where $\mathrm{i}^{\prime} \mathrm{i}^{\prime}<\mathrm{j}^{\prime}<\mathrm{j}, \mathrm{i}^{\prime}-\mathrm{i}+\mathrm{j}-\mathrm{j}^{\prime}>2$. According to equation 2 , it seems that the algorithm should travel all pairs (i',j')in the interval for each step. By utilizing the regularity of energy rule of internal loop, equation 5 splits the contribution to energy $\operatorname{VBI}(i, j)$. The contributions to the energy $\operatorname{VBI}(i, j)$ of an internal loop closed by $(i, j)$ are split into three parts(Lyngso et al, 1999): (a). An entropic term that depends on the size of the loop. (b). Stacking energies for the mismatched base pairs adjacent to the enclosing (exterior and interior) base pair. (c). An asymmetry penalty for asymmetric loops. We write the internal loop energy function as:

$$
\operatorname{VBI}(i, j)=\min _{\substack{i<i^{\prime}<j^{\prime}<j \\
i^{\prime}-i+j-j^{\prime}>2}}\left\{\begin{array}{l}
\operatorname{size}\left(i^{\prime}-i+j-j^{\prime}-2\right)+\operatorname{asymmetry}\left(i^{\prime}-i-1, j-j^{\prime}-1\right) \\
+\operatorname{stacking}(i, j)+\operatorname{stacking}\left(i^{\prime}, j^{\prime}\right)+V\left(i^{\prime}, j^{\prime}\right)
\end{array}\right\}
$$

Lyngso's method consider bulge loop as a special internal loop. However, energy rule for bulge loop is different from internal loop, so bulge loop usually are not counted as special loop. We repartition the contributions to the energy of internal loop. Besides energy depending on size of loop and asymmetry penalty, our method adds bulge loop energy contribution. Stacking energy is not explicitly evaluated as a contribution to energy of internal loop, but a contribution to three parts of energy above. The rest of this section will describe that how our method calculates VBI $(i, j)$ through dividing the dynamic matrix. By equation 5, the energies of bulge loops are easy to be calculated separately by selecting the minimum energy among all pairs energy in the horizontal and vertical line with $(i, j)$. The asymmetry $\left(i^{\prime}-i-1, j-j '-1\right)$ is a energy function depending on $\mid \mathrm{i} '+\mathrm{j}$ '-i-jl (Papanicolaou et al. 1984). As experiment shown, the asymmetry(i'-i-1,jj'-1) reaches a maximum value (MAX_ASY) when li' +j'-i-jl>5 (Papanicolaou et al. 1984). We therefore separately calculate the contribution of the asymmetry(i'-i-1,j-j'1) using equation 5 for $\left|\mathrm{i}^{\prime}+\mathrm{j}^{\prime}-\mathrm{i}-\mathrm{j}\right| \square 5$ (see the region between slash $\mathrm{i}$ and slash $\mathrm{i}+11$ in Figure 1). 


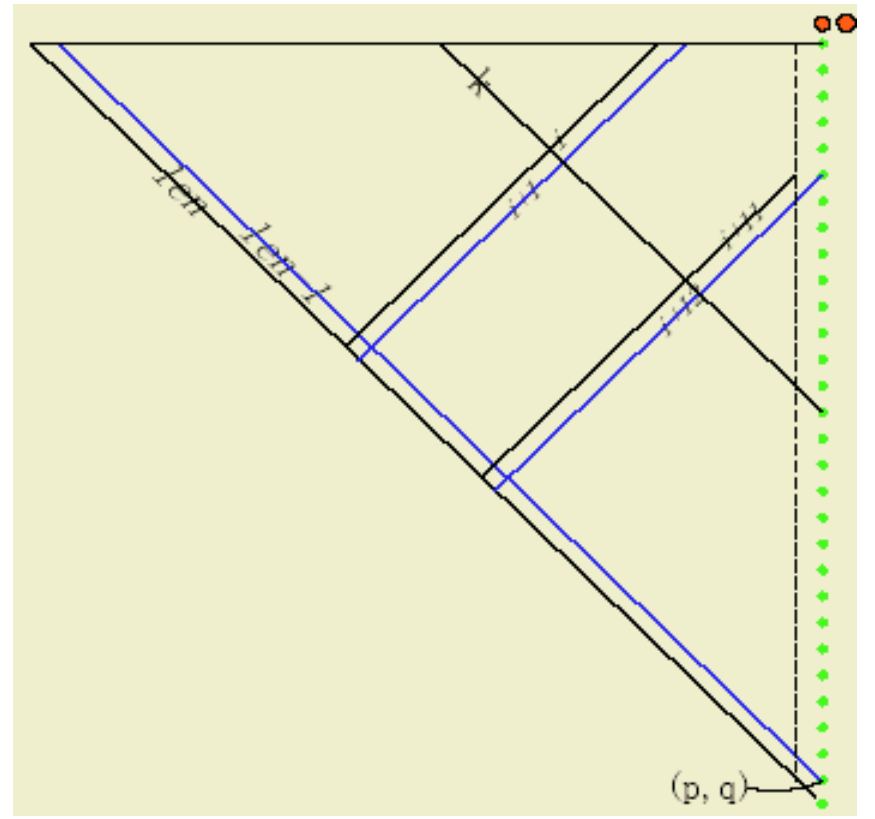

Fig. 1. The spatial correlation between the calculation of $\operatorname{VBI}(i, j)$ and $\operatorname{VBI}(i, j+1)$. From left to right, the two pairs in the right top are $(i, j)$ and $(i, j+1)$ respectively. For VBI $(i, j)$, the region of asymmetry locate between slash $i$ and slash $i+11$. For VBI $(i, j+1)$, the region of asymmetry locate between slash $i+1$ and slash $i+12$. The additional base pairs are the colored pairs located in the rightmost column. This figure doesn't give bulge loops

Now, we can calculate the minimum among all pairs located in all diagonal lines, that is the energy depending on size of loop. After get the minimum energy of asymmetry penalty, we can replace asymmetry(i'-i-1,j-j'-1) in equation 5 by its maximum value(MAX_ASY), thus equation only depending on size of loop is rewritten as:

$V B I \_l e n(i, j)=\min _{\substack{i<i^{\prime}<<<j \\ i^{\prime}-i+j-j^{\prime}>2}}\left\{\begin{array}{l}\operatorname{size}\left(i^{\prime}-i+j-j^{\prime}-2\right)+\operatorname{stacking}(i, j) \\ +\operatorname{stacking}\left(i^{\prime}, j^{\prime}\right)+V\left(i^{\prime}, j^{\prime}\right)+M A X_{-} A S Y\end{array}\right\}$

In fact, most of the pairs traveled in calculating $\operatorname{VBI}(i, j+1)$ have been traveled in calculating VBI $(i, j)$. From $(i, j)$ to $(i, j+1)$, some new pairs $\{(i, k) \mid i<=k<=j+1\}$ (see Figure 1) are added and loop sizes of all pairs (i',j') are increased by 1 . The internal loop size of pairs, which internal loop size is len-l when calculating $\operatorname{VBI}(\mathrm{i}, \mathrm{j})$, is len when calculating $\operatorname{VBI}(\mathrm{i}, \mathrm{j})$. In equation 6 , stacking $\left(i^{\prime}, j^{\prime}\right)+V\left(i^{\prime}, j^{\prime}\right)$ is independent of size of loop. In order to avoid redundant calculation, the minimum energy among the pairs with the same internal loop size is stored for the next step. We add a new array with the size of the internal loop as index and each entry represents the minimum energy among pairs with the same internal loop size. 
$\operatorname{LOOP}($ len $)=\min _{i^{\prime}-i+j-j^{\prime}+2=l e n}\left\{\operatorname{stacking}\left(i^{\prime}, j^{\prime}\right)+V\left(i^{\prime}, j^{\prime}\right)\right\}$

$\operatorname{LOOP}(l e n)$ is the minimum energy among all pairs (i',j') which form loops with size len. Equation 3 only represents the meaning of array LOOP and LOOP(len) can be deduced from $\operatorname{LOOP}($ len- 1$)$ instead of selecting a minimum among the energy of all pair (i',j') using equation 7 . From $(i, j)$ to $(i, j+1)$, some additional base pairs are added. But only one new pair is added to the set of pairs with the same internal loop size or one diagonal line in Figure 1(see Figure 1. One new pair in each diagonal at each

$$
\text { LOOP }(\text { len })=\min \{\operatorname{LOOP}(\text { len }-1), \operatorname{stacking}(p, q)+V(p, q)\}
$$

step). Supposed that the new pair is $(\mathrm{p}, \mathrm{q})$, we get the formulation:

When calculating $\operatorname{VBI}(\mathrm{i}, \mathrm{j})$, the minimum energy is quickly obtained using equation 8 instead of calculating the energy for all pairs. Figure 1 shows the relationship between the calculation of $\operatorname{VBI}(i, j)$ and $\operatorname{VBI}(i, j+1)$. The internal loop size of pairs in diagonal len-1 is len-1 when calculating $\operatorname{VBI}(\mathrm{i}, \mathrm{j})$, however, it becomes len when calculating $\operatorname{VBI}(\mathrm{i}, \mathrm{j}+1)$, then $\operatorname{LOOP}($ len $)$ is updated by formulation 4 . The update of each entry of array LOOP(len) needs only one comparison operation between previous entry LOOP(len-1) and the energy of one new pair such as the colored pair in Figure 1.

Thus the calculation of $\operatorname{VBI}(i, j)$ is divided into three independent part. We rewrite (2) as :

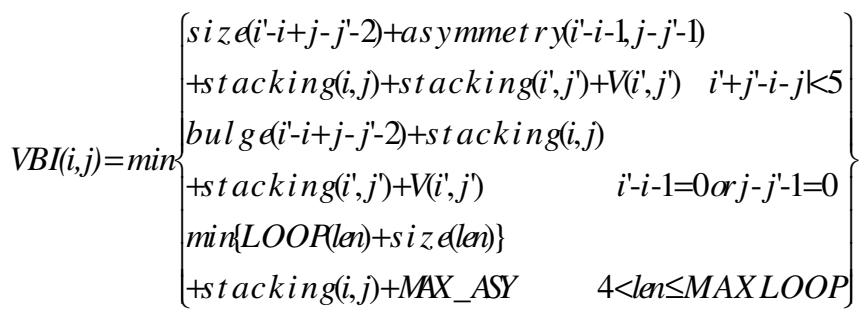

In equation 2, it takes O(MAXLOOP*MAXLOOP/2) to the free energy for the internal loop whereas this computations in equation 9 only takes $\mathrm{O}(\mathrm{MAXLOOP} * \mathrm{C})$, where $\mathrm{C}$ is const value. The time complexity of the original algorithm is $\mathrm{O}\left(\mathrm{n}^{2} *\right.$ MAXLOOP ${ }^{2}$ ) or $\mathrm{O}\left(\mathrm{n}^{4}\right)$ when the length of internal loop is unrestricted. The optimized algorithm reduces the time complexity to $\mathrm{O}\left(\mathrm{n}^{2} *\right.$ MAXLOOP) or $\mathrm{O}\left(\mathrm{n}^{3}\right)$ using only $\mathrm{O}(\mathrm{MAXLOOP})$ or $\mathrm{O}(\mathrm{n})$ space.

Our method reduces the time complexity of dynamic programming algorithm to $\mathrm{O}\left(\mathrm{n}^{3}\right)$ owing to reducing the $\mathrm{O}\left(\mathrm{n}^{4}\right)$ algorithm for calculating internal loops to $\mathrm{O}\left(\mathrm{n}^{3}\right)$. If the size of internal loops is restricted to MAXLOOP(30), the time complexity of internal loops evaluation is $\mathrm{O}\left(\mathrm{n}^{2} *\right.$ MAXLOOP) or $\mathrm{O}\left(\mathrm{n}^{2}\right)$ (MAXLOOP is a constant), however, the evaluation of multi-branched loops is $\mathrm{O}\left(\mathrm{n}^{3}\right)$. The overall time complexity of optimized algorithm is $\mathrm{O}\left(\mathrm{n}^{3}\right)$. 


\section{Implementation}

We implemented the method described here by modifying the open source package ViennaRNA since the package just implements a $\mathrm{O}\left(\mathrm{n}^{3}\right)$ dynamic programming algorithm with restricted size of internal loops (MAXLOOP=30), its time complexity is $\mathrm{O}\left(\mathrm{n}^{4}\right)$ when the size of loops is unrestricted. We call the optimized package IctRNA. The prediction results of IctRNA are the same with that of original ViennaRNA package.

Experimental results show that IctRNA has significant speedup and the platform used is $1.6 \mathrm{GHz}$ Opteron processor, 3GB memory. First, we restrict the maximum size of internal loops to 30. IctRNA is a newly implementation of ViennaRNA using our $\mathrm{O}\left(\mathrm{n}^{3}\right)$ algorithm. Table 1 shows that the time comparison between ViennaRNA and IctRNA. With the length of RNA sequences increasing, the speedup contribution of optimized internal loop decreases because its time contribution to the overall time decreases. For this case, the bottleneck of algorithm is not internal loop but the evaluation of multi-branched loops. In ViennaRNA, when predicting secondary structure for $l s u \_e c o l i$, the time of evaluating multi-branched loops(VM) almost is two times as the time of evaluating internal loops(VBI).

Table 1. The runtime in seconds of ViennaRNA and the IctRNA. VBI: the time of evaluating internal loops

\begin{tabular}{|l|l|l|l|l|}
\hline \multicolumn{1}{|c|}{ programs } & \multicolumn{2}{|c|}{ ViennaRNA } & \multicolumn{2}{c|}{ IctRNA } \\
\hline sequences & VBI & overall & VBI & overall \\
\hline $\begin{array}{l}\text { trna_yeast_phe.fa } \\
\text { (83bp) }\end{array}$ & 0.010 & 0.016 & 0.007 & 0.013 \\
\hline $\begin{array}{l}\text { 5S_ecoli.fa } \\
\text { (154bp) }\end{array}$ & 0.036 & 0.078 & 0.023 & 0.032 \\
\hline $\begin{array}{l}\text { SRP_human.fa } \\
\text { (312bp) }\end{array}$ & 0.401 & 0.454 & 0.217 & 0.262 \\
\hline $\begin{array}{l}\text { RNaseP_ecoli.fa } \\
\text { (387bp) }\end{array}$ & 0.560 & 0.643 & 0.336 & 0.423 \\
\hline $\begin{array}{l}\text { ssu_ecoli.fa } \\
\text { (1556bp) }\end{array}$ & 11.061 & 16.390 & 6.359 & 11.618 \\
\hline $\begin{array}{l}\text { lsu_ecoli.fa } \\
\text { (2918bp) }\end{array}$ & 37.642 & 69.123 & 22.477 & 50.073 \\
\hline
\end{tabular}

We also test the performance of the optimized algorithm with unrestricted size of internal loops. We set the maximum size of internal loops is $n$, where $n$ is the length of RNA sequence. In order to emphasize the effect of optimized algorithm for internal loops calculation, we only compare the time of calculating internal loops and overall time. So the two programs have an improved calculation of multi-branched loops. Table 2 gives experimental results. From Table 2, we can find that a majority of 
overtime is the calculation of internal loops. Our optimized algorithm on the calculation of internal loops achieves huge speedup.

Table 2. The runtime in seconds of ViennaRNA and the optimized algorithm (IctRNA)

\begin{tabular}{|l|l|l|l|l|}
\hline program & \multicolumn{2}{|l|}{ ViennaRNA } & IctRNA \\
\hline sequences & VBI & overall & VBI & owerall \\
\hline $\begin{array}{l}\text { trna_yeast_phe.fa } \\
\text { (83bp) }\end{array}$ & 0.009 & 0.017 & 0.005 & 0.012 \\
\hline $\begin{array}{l}\text { 5S_ecoli.fa } \\
(154 b p)\end{array}$ & 0.075 & 0.083 & 0.019 & 0.028 \\
\hline $\begin{array}{l}\text { SRP_human.fa } \\
\text { (312bp) }\end{array}$ & 3.358 & 3.381 & 0.253 & 0.289 \\
\hline $\begin{array}{l}\text { RnaseP_ecoli.fa } \\
\text { (387bp) }\end{array}$ & 7.359 & 7.412 & 0.251 & 0.523 \\
\hline $\begin{array}{l}\text { ssu_ecoli.fa } \\
(1556 b p)\end{array}$ & 3204.177 & 3208.005 & 31.786 & 35.482 \\
\hline $\begin{array}{l}\text { 1su_ecoli.fa } \\
(2918 b p)\end{array}$ & 51166.314 & 51194.369 & 213.668 & 237.222 \\
\hline
\end{tabular}

Mfold also implements a $\mathrm{O}\left(\mathrm{n}^{3}\right)$ algorithm with the unrestricted size of the internal loops. Obviously, our implementation of $\mathrm{O}\left(\mathrm{n}^{3}\right)$ algorithm is different from Mfold, which is based on the method proposed by Lyngso, R.B. and Zuker, M et al (1999). However, our implementation is faster than Mfold as shown Chart1.

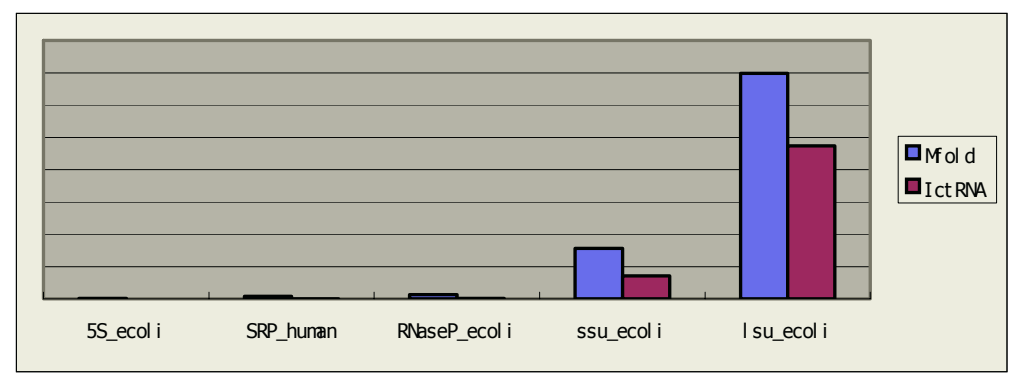

Chart 1. The runtime in seconds of IctRNA and Mfold. The platform used is $1.6 \mathrm{GHz}$ Opteron processor. The overall time of Mfold for 5 sequences (5S_ecoli, SRP_human, RnaseP_ecoli, ssu_ecoli and lsu_ecoli) are $0.796 \mathrm{~s}, 3.987 \mathrm{~s}, 6.746 \mathrm{~s}, 78.112 \mathrm{~s}$ and $349.568 \mathrm{~s}$

\section{Conclusion and Acknowledgements}

The dynamic programming algorithm for RNA secondary structure prediction contains redundant calculation. We take advantage of spatial correlation between two 
continuous steps to eliminate the redundancy and decrease the times of computation in computing internal loops and multi-branched loops energy. The optimized algorithm is much faster than the original program ViennaRNA, we will build a web server based on IctRNA.

We would like to thank Dr.Geir Skogerbo and Dr. Dongbo Bu for revising this paper. This work is supported by National Natural Science Foundation of China under the contract no. 60373044 and knowledge innovative project of Chinese Academy of Sciences under the contract no. KSCX2-SW-233.

\section{Reference}

1. Zuker,M. and Stiegle,P. (1981) Optimal computer folding of larger RNA sequences using thermodynamics and auxiliary information. Nucleic Acids Res., 9, 133-148

2. Lyngso, R. B. and Zuker, M et al (1999) Fast evaluation of internal loops in RNA secondary structure prediction. Bioinformatics. Vol.15(6), 440-445

3. HoHacker,IvoL.(1994) http:// www. tbi. univie. ac. at/ ivo /RNA/

4. Hofacker, Ivo L. (2003)Vienna RNA secondary structure server. Bioinformatics. Vol.31 (13), 3429-3431, 2003

5. Tinoco,I., Uhlenbeck, O.C. and Levine, M.D.(1971) Estimation of secondary structure in ribonucleic acids. Nature, 230, 362-367.

6. Tinoco,I and Borer, N, P et al(1973) Improved estimation of secondary structure in ribonucleic acids. Nature New Biology, 246:40-41

7. Rune B.Lyngs $\varnothing$, Michael Zuker and Christian N. S. Pedersen. (1999) An Improved Algorithm for RNA Secondary Structure Prediction. BRICS May 1999

8. Michael Zuker. (2003) Mfold web server for nucleic acid folding and hybridization prediction. Nucleic Acids Res. 31 (13), 3406-15, 2003

9. Papanicolaou, C., Gouy,M. and Ninio, J. (1984) An energy model that predicts the correct folding of both the tRNA and the 5S RNA molecules. Nucleic Acids Res., 12, 31-44.

10. Waterman,M.S. and Smith,T.F. (1986) Rapid dynamic programming methods for RNA secondary structure. Adv. Appl. Math., 7, 455-464. 